\title{
Development of the Canadian COVID-19 Emergency Department Rapid Response Network population-based registry: a methodology study
}

\author{
Corinne M. Hohl MD MHSc, Rhonda J. Rosychuk PhD, Andrew D. McRae MD PhD, \\ Steven C. Brooks MD MHSc, Patrick Archambault MD MSc, Patrick T. Fok MDCM PhD, \\ Philip Davis MD MSc, Tomislav Jelic MD, Joel P. Turner MD MSc, Brian H. Rowe MD MSc, \\ Éric Mercier MD MSc, Ivy Cheng MD PhD, John Taylor MD MPH, Raoul Daoust MD MSc, \\ Robert Ohle MBBCh MSc, Gary Andolfatto MD, Clare Atzema MD MSc, Jake Hayward MD MPH, \\ Jaspreet K. Khangura MD MSc, Megan Landes MD MSc, Eddy Lang MD, Ian Martin MD MHSc,
} Rohit Mohindra MD MASc, Daniel K. Ting MD, Samuel Vaillancourt MD MPH, Michelle Welsford MD, Baljeet Brar MD, Tara Dahn MD MASc, Hana Wiemer MD, Krishan Yadav MD MSc, Justin W. Yan MD MSc, Maja Stachura MD, Colleen McGavin BA, Jeffrey J. Perry MD MSc, Laurie J. Morrison MD MSc; for the Canadian COVID-19 Emergency Department Rapid Response Network investigators and for the Network of Canadian Emergency Researchers and the Canadian Critical Care Trials Group

\section{Abstract}

Background: Emergency physicians lack high-quality evidence for many diagnostic and treatment decisions made for patients with suspected or confirmed coronavirus disease 2019 (COVID-19). Our objective is to describe the methods used to collect and ensure the data quality of a multicentre registry of patients presenting to the emergency department with suspected or confirmed COVID-19.

Methods: This methodology study describes a population-based registry that has been enrolling consecutive patients presenting to the emergency department with suspected or confirmed COVID-19 since Mar. 1, 2020. Most data are collected from retrospective chart review. Phone follow-up with patients at 30 days captures the World Health Organization clinical improvement scale and contextual, social and cultural variables. Phone follow-up also captures patient-reported quality of life using the Veterans Rand 12-Item Health Survey at 30 days, 60 days, 6 months and 12 months. Fifty participating emergency departments from 8 provinces in Canada currently enrol patients into the registry.

Interpretation: Data from the registry of the Canadian COVID-19 Emergency Department Rapid Response Network will be used to derive and validate clinical decision rules to inform clinical decision-making, describe the natural history of the disease, evaluate COVID-19 diagnostic tests and establish the real-world effectiveness of treatments and vaccines, including in populations that are excluded or underrepresented in clinical trials. This registry has the potential to generate scientific evidence to inform our pandemic response, and to serve as a model for the rapid implementation of population-based data collection protocols for future public health emergencies. Trial registration: Clinicaltrials.gov, no. NCT04702945

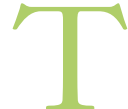
he coronavirus disease 2019 (COVID-19) pandemic is the largest public health crisis in over a century. ${ }^{1}$ As of Jan. 15, 2021, COVID-19 has resulted in over 113 million infections and almost 2.5 million deaths globally. ${ }^{2}$ The global crude mortality rate among patients diagnosed with COVID-19 is about $3 \%$, but some countries have reported rates that are up to 3 times higher. ${ }^{2,3}$ Factors explaining these variations include population differences in demographics, health status and socioeconomics, as well as system factors such as the availability of testing, pandemic preparedness and response, with others yet to be uncovered. 4,5 There is an urgent need for high-quality, populationlevel data to understand modifiable risks for disease severity
Competing interests: Patrick Fok is a shareholder of Hologic, Merck Pharmaceuticals and Moderna. Brian Rowe is the Scientific Director of the Institute of Circulatory and Respiratory Health at the Canadian Institutes of Health Research (ClHR) and reports grants and salary from the CIHR outside the submitted work. Hana Wiemer reports grants and nonfinancial support from Purdue Pharma Canada outside the submitted work. Justin Yan reports grants from Government of Ontario Ministry of Colleges and Universities, during the conduct of the study. No other competing interests were declared.

This article has been peer reviewed.

Correspondence to: Corinne Hohl, corinne.hohl@ubc.ca

CMAJ Open 2021. DOI:10.9778/cmajo.20200290 
and transmissibility, to evaluate therapies and vaccines and to develop evidence-based prevention, treatment and resource allocation strategies.

The emergency department is often the first point of contact for patients with severe COVID-19, and is the location where critical decisions regarding management and disposition are made. ${ }^{6}$ These decisions affect downstream health resource use and transmissibility. Early in the pandemic, emergency physicians were encouraged to intubate hypoxic patients early in their presentation to reduce aerosols, based on theoretical considerations without evidence of effectiveness. ${ }^{7}$ This and other strategies to manage hypoxia have since evolved, while maintaining good patient outcomes. ${ }^{8}$ Similarly, early, poor-quality data showed a possible benefit of hydroxychloroquine, now proven ineffective. ${ }^{9}$

We developed the Canadian COVID-19 Emergency Department Rapid Response Network (CCEDRRN) to collect high-quality, population-based data from geographically distributed sites over the course of the pandemic. We will use this registry to derive and validate clinical decision rules to enable evidence-based emergency department decisionmaking. We will also use the registry to evaluate emerging therapies and vaccines, particularly among populations commonly excluded or underrepresented in clinical trials. Our objective is to describe the methods we have used to collect and ensure the data quality in our registry of patients suspected and confirmed to have COVID-19.

\section{Methods}

\section{Study design and setting}

This methodology study outlines the development of the CCEDRRN registry. We designed this population-based, multisite registry to enrol consecutive eligible patients presenting with suspected or confirmed COVID-19 to 50 emergency departments in 8 Canadian provinces, from Mar. 1, 2020 onward (Figure 1; for details on contributing sites and study investigators, see Appendix 1, available at www.

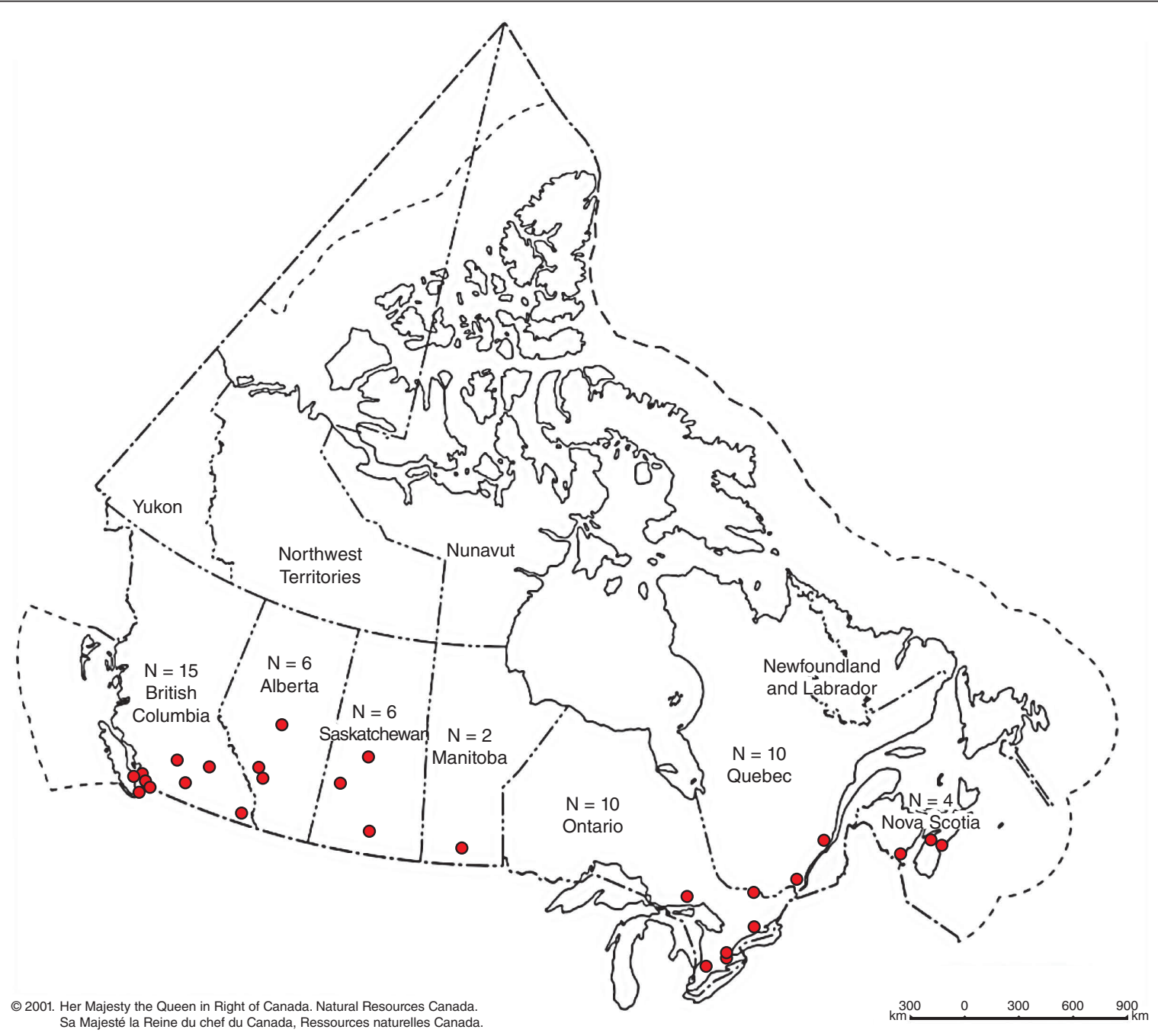

Figure 1: Participating sites of the Canadian COVID-19 Emergency Department Rapid Response Network registry. This figure contains information licensed under the Open Government Licence - Canada (https://open.canada.ca/en/open-government-licence-canada). 
cmajopen.ca/content/9/1/E261/suppl/DC1). We maintain a list of network investigators and hospitals on our website (https://canadiancovid19ednetwork.org). ${ }^{10}$

\section{Study population}

We are enrolling patients presenting to participating emergency departments with suspected or confirmed COVID-19. We defined 2 periods for enrolment based on the availability of COVID-19 testing (Table 1). Research assistants use medical microbiology testing and discharge diagnoses to screen for potentially eligible patients (Table 2).

In Period 1, when testing for COVID-19 in each province was restricted to specific patient populations (e.g., health care workers, admitted patients), we included patients meeting the World Health Organization (WHO) criteria for suspected COVID-19 (i.e., fever and a respiratory symptom, such as shortness of breath) when they visited the emergency department, and those who tested positive for severe acute respiratory syndrome coronavirus 2 (SARS-CoV-2) in emergency departments. ${ }^{11}$ Early case series showed that fever may be absent in many patients with COVID-19. ${ }^{12}$ Therefore, we were liberal in our interpretation of fever, and included patients with self-reported or subjective fever. In addition, all sites screened for cases by reviewing the charts of patients with relevant discharge diagnoses or, when this was not available, presenting complaints (Table 2). Period 1 had no exclusion criteria.

Period 2 started on the date on which each province expanded testing criteria, allowing clinicians to test patients based on clinical suspicion or policy. In Period 2, we are including patients who were tested for SARS-CoV-2 in the emergency department or within 24 hours of arrival. We are also including patients presenting with a test that was confirmed positive for SARS-CoV-2 from the community or another facility, and those diagnosed with a complication related to COVID-19 (Table 2). We are excluding patients tested for SARS-CoV-2 in the context of an elective admission (e.g., planned hip revision) and those seen in the emergency department directly by another service (e.g., trauma team activation).

\section{Data collection by site}

By Sept. 21, 2020, only 4\% of patients meeting inclusion criteria were testing positive for SARS-CoV-2 (Gelareh Ghaderi, CCEDRRN: unpublished data, 2020), thereby providing the registry with a high volume of controls testing negative for SARS-CoV-2. We thus redirected the network to accrue a larger sample of patients confirmed to have SARS-CoV-2 to provide us with greater power for longitudinal comparison studies, and to enable clinical decision rule development with risk stratification of patients with COVID-19.

We transitioned sites with high-volume data collection and low positivity rates for SARS-CoV-2 ( $<2 \%$ test positivity) to instead collect data on consecutive COVID-19 cases only (COVID-19 data collection sites). These sites establish consecutive COVID-19 cases based on positive test results from specimens taken for the nucleic acid amplification test in the emergency department, within 24 hours of arrival, or the first 14 days of hospitalization. These sites also capture patients with clinical symptoms of COVID-19 presenting to the emergency department within 14 days of receiving test results positive for COVID-19.

At sites with adequate human resources and a higher incidence of SARS-CoV-2 (i.e., $\geq 2 \%$ test positivity rate), we continue to capture data on both patients with suspected (negative test results) and confirmed (positive test results) COVID-19 (full data collection sites).

Table 1: Screening date, province and inclusion criteria

\section{Period 1}

- Alberta: Mar. 1-Apr. 7, 2020

- British Columbia: Mar. 1-Apr. 19, 2020

- Manitoba: Mar. 1-Apr. 27, 2020

- New Brunswick: Mar. 1-Apr. 12, 2020

- Nova Scotia: Mar. 1-Apr. 5, 2020

- Ontario: Mar. 1-May 13, 2020

- Quebec: Mar. 1-May 3, 2020

- Saskatchewan: Mar. 1-Apr. 2, 2020

\section{Period 2}

- Alberta: Apr. 8, 2020 onward

- British Columbia: Apr. 20, 2020 onward

- Manitoba: Apr. 28, 2020 onward

- Nova Scotia: Apr. 6, 2020 onward

- New Brunswick: Apr. 13, 2020 onward

- Ontario: May 14, 2020 onward

- Quebec: May 4, 2020 onward

- Saskatchewan: Apr. 3, 2020 onward

\section{Criteria}

- Presenting to the ED meeting WHO clinical criteria for suspected COVID-19:

- Fever and

- Respiratory syndrome, including flu-like illness, shortness of breath or cough

- Presenting to the ED and tested for SARS-CoV-2 in the ED

Criteria

- Tested for SARS-CoV-2 in the ED or within 24 hours of arrival

- Elective, non-ED admissions excluded

- Patient presenting to the ED within 14 days of a positive SARS-CoV-2 test and presenting with clinical symptoms of COVID-19.

Note: COVID-19 = coronavirus disease 2019, ED = emergency department, SARS-CoV-2 = severe acute respiratory syndrome coronavirus 2 , WHO = World Health Organization 


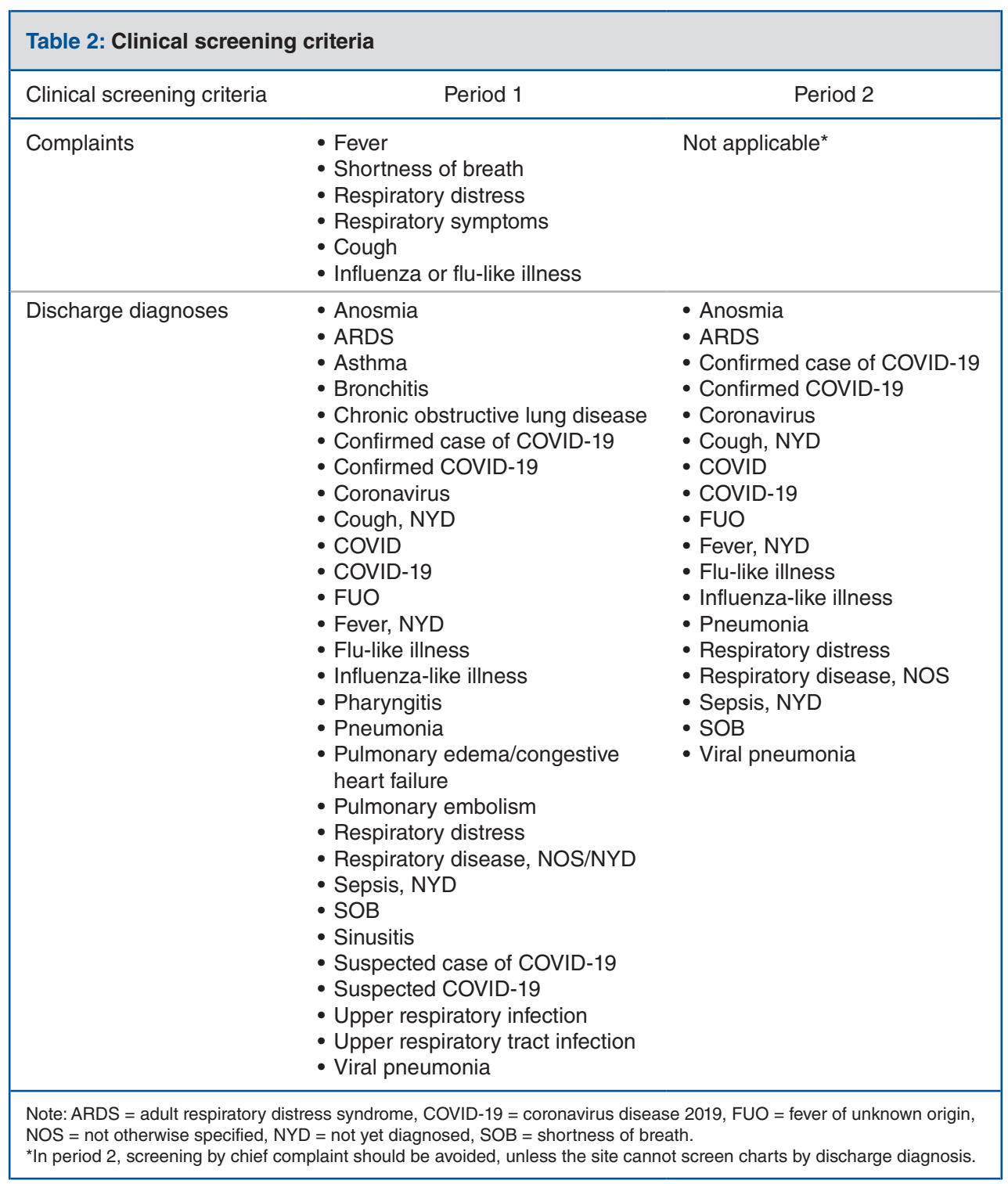

\section{Data sources}

We developed standardized operating procedures for screening potentially eligible patients for COVID-19 and for full data collection sites, and standardized data entry and followup across the network.

A national research coordinator and provincial research coordinators drafted, piloted, refined and subsequently oversaw the implementation of standard operating procedures for data collection and the study conduct. The research coordinators all have extensive research experience in emergency medicine or related fields and expertise in data abstraction. The national research coordinator onboards all research assistants remotely and uses instructional videos to ensure consistency in data collection across the network. Provincial coordinators assist with data verification, quality checks and study coordination. Research assistants complete all institutional privacy training requirements.

\section{Retrospective data source}

Retrospective data is collected by research assistants, who abstract data from medical charts and enter it into a central, Web-based REDCap database (Vanderbilt University). They conduct data abstraction at 30 days after the index visit to the emergency department. This schedule captures additional emergency department hospital admissions and deaths. We keep current data dictionaries on our website (Appendix 2, available at www.cmajopen.ca/content/9/1/ E261/suppl/DC1). ${ }^{10}$

\section{Prospective data source}

Physicians from emergency departments at the initial data collection sites (see Data quality section for details) completed a prospective data collection of 32 important clinical data points, enabling us to evaluate missing data and reliability of retrospective data collection (prospective data collection sheet 
available in Appendix 3, available at www.cmajopen.ca/content/ 9/1/E261/suppl/DC1).

\section{Telephone follow-up}

At 30 days after the index visit to the emergency department, we contact patients by telephone to obtain verbal consent for follow-up. We measure the Veterans Rand 12-Item Health Survey (VR12), ${ }^{13-15}$ calculate the WHO Ordinal Outcome Scale $^{16}$ and ask questions regarding culture, race, ethnicity, occupation, socioeconomics and gender, as well as self-isolation. We had developed these questions previously with input from people with lived experience with COVID-19. We repeat VR12 assessments at 60 days, 6 months and 12 months after the index visit. In January 2021, we added vaccination status to our follow-up questionnaires. We piloted the follow-up tool in British Columbia, Ontario and Nova Scotia (see followup data collection form at Appendix 4, available at www. cmajopen.ca/content/9/1/E261/suppl/DC1).

It is not feasible to follow up all suspected patients enrolled at full data collection sites. For every patient with COVID-19, we randomly select 4 control patients who tested negative for COVID-19 (suspected COVID-19) for every COVID-19 case from the same site and date for follow-up, as little gains in power occur for more than 4 controls per case. ${ }^{17}$ If a control patient cannot be reached, we randomly select control patients until we successfully reach 4 control patients.

\section{National administrative data sources}

The Health Data Research Network facilitated the development of a data flow of personal health identifiers and study identification numbers from each province allowing us to link registry data with national administrative data repositories (Figure 2).

\section{Data quality}

We developed a data monitoring protocol to document the process of data verification and editing, and to outline targets for data completeness. We programmed internal logic and error checks in REDCap to ensure that nonsensical values could not be entered (e.g., an admission date preceding the visit to the emergency department). An analyst completes biweekly data quality checks to identify missing, incomplete and outlying data, and returns records to sites for completion and verification or editing. Missing data are measured monthly and continue to be less than $1 \%$ for highly important data points. We embedded free text fields into data collection instruments for when subjective assessments are required or no uniform data standard exists. A qualitative research assistant reviews text data iteratively to refine data fields and data dictionaries, and developed explanatory notes in REDCap to optimize data collection.

For the first months of enrolment, we measured the interrater agreement between variables collected prospectively and retrospectively on 811 cases (Table 3). We terminated prospective data collection after showing the reliability of retrospective data capture.

\section{Outcomes}

In accordance with the WHO case definition at the time we created the registry, we define "suspected COVID-19" as a patient with fever and at least 1 symptom or sign of respiratory illness (e.g., cough, shortness of breath or flu-like illness); a patient with an epidemiologic link to COVID-19 infection, including travel to an affected area within the past 14 days, local community spread or contact with a confirmed or probable case of COVID-19; or a patient with no alternative diagnosis that fully explains the clinical presentation. ${ }^{18}$

We define "confirmed COVID-19" as any patient in whom a biological specimen tested positive for SARS-CoV-2 using the nucleic acid amplification test. The specimen had to have been drawn within 2 weeks of the emergency department visit, if the patient presented to the emergency department with a complication related to COVID-19. If the initial test result was negative, the patient had to have a specimen that tested positive within 14 days after the index visit, accounting for the longest possible incubation period.

Additional outcome variables include admission, mechanical ventilation, subsequent emergency department visits, readmissions, death, discharge from hospital, clinical recovery and quality of life. Each writing group will select the most appropriate outcome for its study question.

\section{Data management}

After assigning a unique study identifier, deidentified data are collected and stored using REDCap at the University of British Columbia. After verification, registry data are uploaded into CaraSpace, a secure, private cloud for storage and analysis of privacy-sensitive data (https://www.popdata.bc.ca/ secure_data/CaraSpace). Analysts access the cloud space via an encrypted Virtual Private Network through a firewall and 2-factor authentication. This process enables remote data access and sharing without transfer of data, minimizing privacy risks.

\section{Governance}

The CCEDRRN steering committee consists of a chair, vicechair, a site investigator from each participating site and patient partners with lived experience of COVID-19 (Figure 3). Member investigators can propose additional retrospective, prospective or follow-up data collection to answer emerging research questions. Members and external investigators can apply to CCEDRRN's Protocol Review and Publication and Data Access and Management Committees for access to data.

\section{Statistical analysis}

Each manuscript writing group will develop an analytic protocol for its specific study question, which will be reviewed for appropriateness by CCEDRRN's Protocol Review and Publication Committee. Each group will ensure that missing data and potential confounders be addressed for each study question. We use the Cohen $\kappa$ statistic to measure the interrater agreement of variables collected both prospectively and retrospectively. ${ }^{19}$ 


\section{cmaJOPEN}

Research

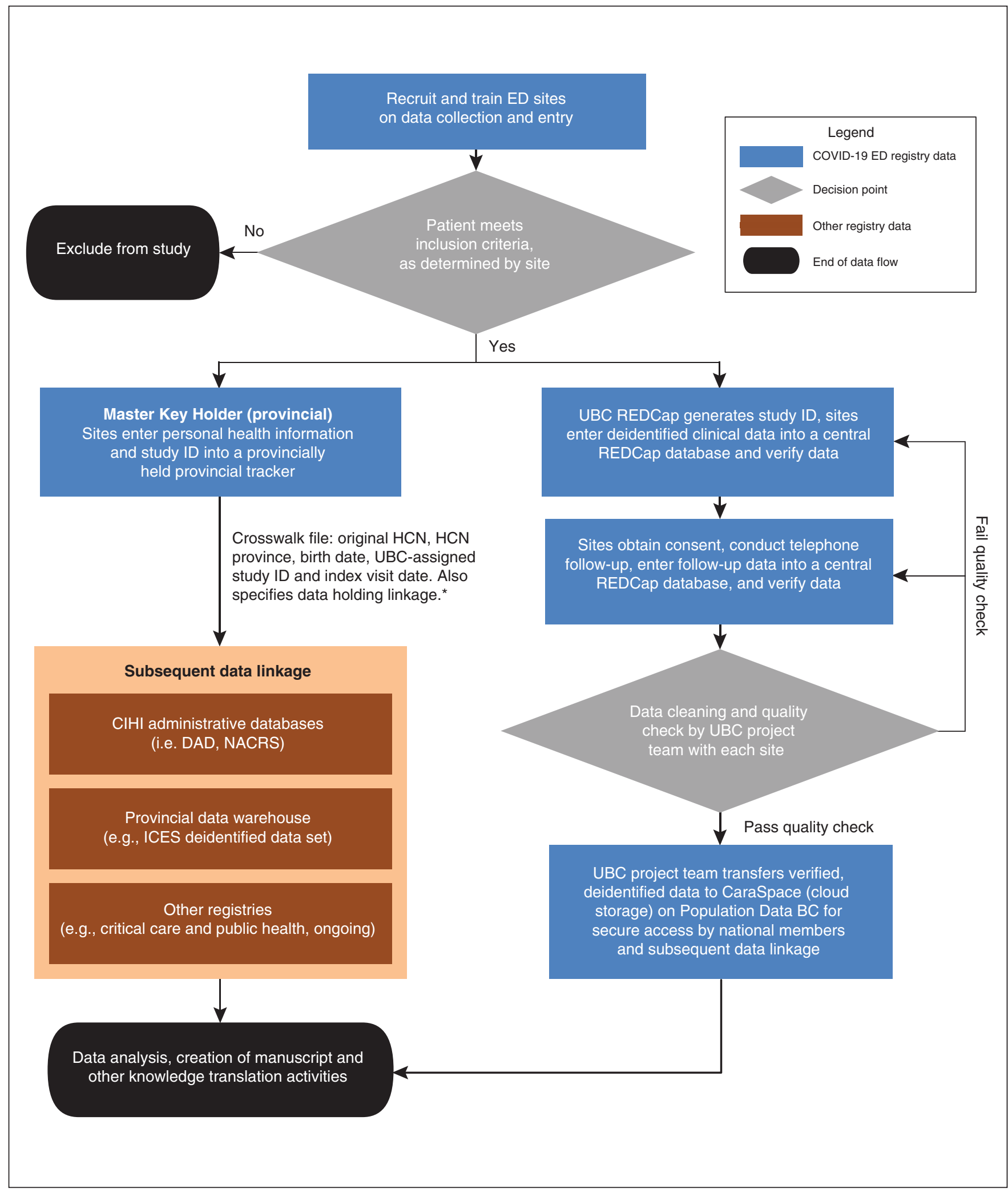

Figure 2: Data flow diagram for registry data. Note: $\mathrm{CIHI}=$ Canadian Institute for Health Information, COVID-19= coronavirus disease 2019 , $\mathrm{DAD}=$ Discharge Abstract Database, ED = emergency department, $\mathrm{HCN}=$ health care number, ID = identification number, NACRS = National Ambulatory Care Reporting System, UBC = University of British Columbia. ${ }^{*}$ The Protocol Review and Publication Committee reviews the Registry Study manuscript proposal and recommends to the Registry Executive Committee (REC) that it is in scope. The Data Access and Management Committee reviews and recommends to the REC that all the necessary agreements and approvals are in place to access the data. The REC then approves all manuscripts and data access, including linkage, when it is required. 
Table 3: Interrater agreement between variables collected prospectively and retrospectively from 811 patients

\begin{tabular}{|c|c|}
\hline Variable & $\begin{array}{c}\text { Cohen } \kappa \text { coefficient } \\
(95 \% \mathrm{Cl})\end{array}$ \\
\hline \multicolumn{2}{|l|}{ Living situation } \\
\hline Home, long-term care, homeless, other & 0.76 (0.69 to 0.84$)$ \\
\hline \multicolumn{2}{|l|}{ Symptoms } \\
\hline Cough & $0.63(0.57$ to 0.68$)$ \\
\hline Shortness of breath & $0.67(0.61$ to 0.72$)$ \\
\hline Fever & $0.65(0.60$ to 0.71$)$ \\
\hline Headache & 0.58 (0.51 to 0.66$)$ \\
\hline Nausea or vomiting & $0.53(0.45$ to 0.61$)$ \\
\hline Diarrhea & $0.63(0.55$ to 0.71$)$ \\
\hline Myalgias & $0.40(0.32$ to 0.49$)$ \\
\hline Dysgeusia or anosmia & $0.37(0.11$ to 0.64$)$ \\
\hline \multicolumn{2}{|l|}{ Infection risk } \\
\hline Travel & $0.31(0.04$ to 0.58$)$ \\
\hline Institutional exposure & 0.51 (0.36 to 0.66$)$ \\
\hline Health care worker & $0.69(0.59$ to 0.80$)$ \\
\hline Household or caregiver contact & $0.37(0.19$ to 0.56$)$ \\
\hline Other & 0.24 (0.04 to 0.44$)$ \\
\hline \multicolumn{2}{|l|}{ Comorbidities } \\
\hline Congestive heart failure & 0.71 (0.61 to 0.82$)$ \\
\hline Coronary artery disease & 0.51 (0.39 to 0.62$)$ \\
\hline Hypertension & $0.70(0.64$ to 0.76$)$ \\
\hline Asthma & 0.80 (0.72 to 0.87$)$ \\
\hline Pulmonary fibrosis & $0.39(-0.15$ to 0.94$)$ \\
\hline Chronic lung disease (not asthma or IPF) & $0.72(0.64$ to 0.80$)$ \\
\hline Chronic kidney disease & 0.73 (0.63 to 0.84$)$ \\
\hline Dialysis & $0.58(0.14$ to 0.30$)$ \\
\hline Diabetes & 0.69 (0.61 to 0.77$)$ \\
\hline Liver disease & $0.43(0.17$ to 0.68$)$ \\
\hline Organ transplant & $0.77(0.51$ to 1.00$)$ \\
\hline Chronic neurological disorder (not dementia) & $0.17(-0.01$ to 0.34$)$ \\
\hline Dementia & $0.51(0.26$ to 0.77$)$ \\
\hline Rheumatologic disorder & 0.39 (0.18 to 0.60$)$ \\
\hline Active malignant neoplasm & 0.55 (0.41 to 0.69$)$ \\
\hline Past malignant neoplasm & 0.23 (0.07 to 0.38$)$ \\
\hline Obesity (clinical impression) & $0.22(0.05$ to 0.39$)$ \\
\hline \multicolumn{2}{|l|}{ Respiratory distress } \\
\hline Respiratory distress & $0.18(0.12$ to 0.25$)$ \\
\hline \multicolumn{2}{|l|}{ Other risk factors } \\
\hline Smoking (never, current, past use)* & 0.73 (0.66 to 0.80$)$ \\
\hline Alcohol misuse (never, current, past use) ${ }^{*}$ & $0.53(0.43$ to 0.63$)$ \\
\hline Illicit substance use (never, current, past use)* & $0.82(0.75$ to 0.89$)$ \\
\hline
\end{tabular}

\section{Ethics approval}

This protocol has been approved by the University of British Columbia Research Ethics Board (UBC REB H20-01015) and by participating sites. The protocol was approved with a waiver of informed consent for enrolment, retrospective data collection and storage of Personal Health Information for linkage with administrative data (Figure 2). We obtain verbal consent from patients at the time of first telephone follow-up to collect followup data at 30 and 60 days, and at 6 and 12 months.

\section{Interpretation}

Our network harmonized data collection for patients with suspected and confirmed COVID-19 to enhance research capacity in Canada. This has enabled rapid accrual of high-quality, representative data from across the country to answer priority research questions about COVID-19. As of Feb. 26, 2021, there were 59197 suspected cases and 12378 confirmed COVID-19 cases enrolled into the registry. We will continue enrolling cases as the pandemic evolves.

Registry data are currently being used to derive and validate clinical decision rules, evaluate diagnostic tests, determine the impact of new treatments and vaccinations and complete observational studies as the pandemic evolves. It is anticipated that the registry will enable observational cohort studies on COVID-19.

The creation of this large network was facilitated by rapid mobilization of funding through the Canadian Institutes of Health Research and other agencies, and by a commitment to an open and fair governance structure that enabled all investigators to participate in governance and authorship, guided by the International Committee of Medical Journal Editors criteria (http:// www.icmje.org/). We endorsed the central tenets of the WHO Knowledge Translation and Open Science Frameworks to optimize collaboration and use of our data, including by external investigators..$^{20,21}$ This model of collaboration may be expanded to other disciplines and countries, and for other emerging health crises.

The registry continues to collect data on both suspected and confirmed cases of COVID-19, allowing us to develop and validate clinical decision rules and evaluate diagnostic tests and vaccinations over the course of the pandemic. By collecting data on patients with respiratory syndromes not caused by SARS-CoV-2, we limit attribution bias, which is a common problem in early case series. ${ }^{4,7,8}$

Our broad inclusion criteria and the diversity of participating institutions enable us to collect data on patients commonly excluded from clinical trials, such as First Nations people, prisoners, people who are pregnant or children. Understanding COVID-19 in these vulnerable populations will be important for containing and mitigating the effects of the pandemic. ${ }^{22}$ By collecting data on these patients, we hope 


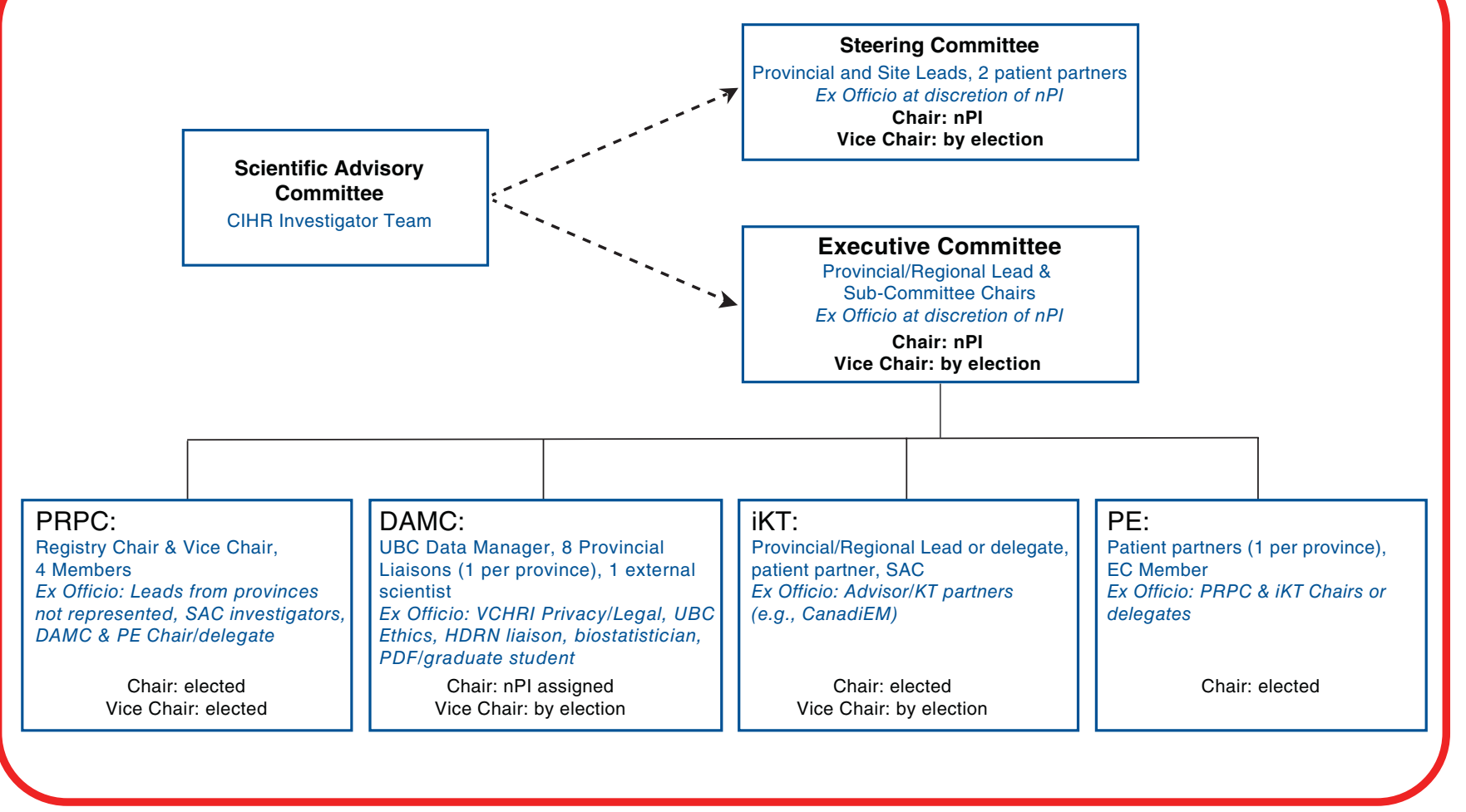

Figure 3: Governance of the Canadian COVID-19 Emergency Department Rapid Response Network. Note: ClHR = Canadian Institutes of Health Research, DAMC = Data Access and Management Committee, EC = Executive Committee, HDRN = Health Data Research Network, $\mathrm{iKT}=$ integrated knowledge translation, $\mathrm{KT}=$ knowledge translation, $\mathrm{nPI}=$ nominated principal investigator, $\mathrm{PE}=$ patient engagement, $\mathrm{PDF}=$ postdoctoral fellow, PRPC = Protocol Review and Publication Committee, SAC = Scientific Advisory Committee, UBC $=$ University of British Columbia, VCHRI = Vancouver Coastal Health Research Institute.

to contribute to the body of evidence required to identify and address gaps in care and policy, and answer questions about COVID-19 in vulnerable patient groups who often seek care in emergency departments. ${ }^{23,24}$

We faced substantial impediments to rapidly mobilize the network, given a nonharmonized approach to research ethics reviews, institutional differences in the interpretation of privacy laws, and a focus on protection of the individual institution. Some provinces opted out of the network or restricted the flow of health data into national repositories. These structural impediments are substantial and need to be addressed urgently through harmonization of provincial privacy law interpretation, a national ethics review process and standardized agreements for interinstitutional data sharing and transfer of funds.

Our integrated knowledge translation plan engages knowledge users and patient partners in defining, refining and prioritizing research questions and study outcomes, and in developing knowledge translation tools and strategies. Although our website (https://canadiancovid19ednetwork.org) is our central knowledge dissemination tool, we have partnered with knowledge translation specialists, including editors of open-access podcasts (e.g., EMcases, https://emergencymedicinecases. com/), infographics (e.g., CanadiEM, https://canadiem.org/) and COVID-19 town halls (hosted by the Canadian Association of Emergency Physicians) to ensure timely and broad dissemination of our research results.

\section{Limitations}

Registry data are based mainly on retrospective chart review and follow-up interviews with patients, which can be limited in quality and quantity. Follow-up interviews are also subject to recall bias. Although prospective and retrospective interrater reliability was moderate to high for most variables, some had poor agreement. We have maximized data quality through standardized procedures, data validation and data quality and logic checks. We have mitigated sources of error and bias by ensuring enrolment of consecutive cases. We anticipated difficulties in collecting follow-up data from disadvantaged populations; we mitigated this by having broad geographical coverage and linkage to national administrative databases to address gaps. Race and ethnicity could not be captured in retrospective data, and can only be collected from patients during follow-up.

Although our network does not include all Canadian provinces and territories, we have included 50 academic and nonacademic sites, including in rural and remote areas, across 8 provinces. Substantial delays in institutional reviews resulted in delays to deriving and validating clinical decision rules 
during the first wave of the pandemic. Fortunately, all sites were enrolling by Aug. 31, 2020, and our first research results are expected in early 2021.

\section{Conclusion}

This protocol describes the harmonized, high-quality collection of data from patients presenting to Canadian emergency departments with suspected and confirmed COVID19 to enhance research capacity during the pandemic. This represents the latest and largest collaborative emergency medicine network in Canada. It has the potential to generate scientific evidence to inform our pandemic response, and to serve as a model for the rapid implementation of population-based data collection protocols for future public health emergencies.

\section{References}

1. Faust JS, Lin Z, Del Rio C. Comparison of estimated excess deaths in New York City during the COVID-19 and 1918 influenza pandemics. $7 A M A$ Netw Open 2020;3:e2017527.

2. WHO coronavirus disease (COVID-19) dashboard. Geneva: World Health Organization. Available: https://covid19.who.int/? gclid=CjwKCAjw5Kv7B RBSEiwAXGDEle_dyJCA3TPMvUjiKBkpY-74Ilp0Nx5ES1iXIGCGfkTnR g8i9YgKhhoCAZIQAvD_BwE (accessed 2021 Mar. 2).

3. COVID-19 map FAQs. Baltimore (MD): Johns Hopkins University Center for Systems Science and Engineering; updated 2020 Dec. 1. Available: https:// systems.jhu.edu/research/public-health/2019-ncov-map-faqs/ (accessed 2020 Mar. 30).

4. Goyal P, Choi JJ, Pinheiro LC, et al. Clinical characteristics of COVID-19 in New York City. N Engl 7 Med 2020;382:2372-4.

5. Botly LCP, Martin-Rhee M, Kasiban A, et al. COVID-19 pandemic: global impact and potential implications for cardiovascular disease in Canada. C7C Open 2020;2:265-72.

6. Berlin DA, Gulick RM, Martinez FJ. Severe COVID-19. N Engl f Med 2020;383:2451-60

7. Yao W, Wang T, Jiang B, et al. Emergency tracheal intubation in 202 patients with COVID-19 in Wuhan, China: lessons learnt and international expert recommendations. Br 7 Anaesth 2020;125:e28-37.

8. Mitra AR, Fergusson NA, Lloyd-Smith E, et al. Baseline characteristics and outcomes of patients with COVID-19 admitted to intensive care units in Vancouver, Canada: a case series. CMA7 2020;192:E694-701.

9. RECOVERY Collaborative Group; Mafham M, Linsell L, Bell JL, et al. Effect of hydroxychloroquine in hospitalized patients with COVID-19. N Engl f Med 2020;383:2030-40.

10. Canadian COVID-19 Rapid Response Network (CCEDRRN). Canadian COVID-19 ED Network. ED COVID-19 Registry; 2020. Available: https:// canadiancovid19ednetwork.org (accessed 2020 Oct. 19).

11. Coronavirus disease 2019 (COVID-19): situation report - 66. Geneva: World Health Organization; 2020. Available: www.who.int/docs/default-source/ coronaviruse/situation-reports/20200326-sitrep-66-covid-19.pdf?sfvrsn=9e 5b8b48_2 (accessed 2020 Mar. 27).

12. Guan $\bar{W}$-J, Ni Z-Y, Hu Y, et al. China Medical Treatment Expert Group for Covid-19. Clinical characteristics of coronavirus disease 2019 in China. NEngl 7 Med 2020;382:1708-20.

13. VR-12. Code Technology. Available: www.codetechnology.com/vr-12-gen eral-health-pro-tool/ (accessed 2020 Sept. 4).

14. Iqbal SU, Rogers S, Selim A, et al. The Veterans RAND 12 Item Health Survey (Vr-12): what it is and how it is used. Boston: Boston University; 2007. Available: www.bu.edu/sph/files/2015/01/veterans_rand_12_item_health_survey_vr-12 2007.pdf (accessed 2020 July 17).

15. Selim AJ, Rogers W, Fleishman JA, et al. Updated U.S. population standard for the Veterans RAND 12-item Health Survey (VR-12). Qual Life Res 2009; $18: 43-52$

16. WHO R\&D Blueprint novel coronavirus: COVID-19 therapeutic trial synopsis. Geneva: World Health Organization; 2020. Available: www.who.int/blue print/priority-diseases/key-action/COVID-19_Treatment_Trial_Design_ Master_Protocol_synopsis_Final_18022020.pdf (accessed 2020 Oct. 28).

17. Taylor JM. Choosing the number of controls in a matched case-control study, some sample size, power and efficiency considerations. Stat Med 1986;5:29-36.

18. Coronavirus disease 2019 (COVID-19): Situation Report - 88. Geneva: World Health Organization; 2020. Available: www.who.int/docs/default-source/ coronaviruse/situation-reports/20200417-sitrep-88-covid-191b6cccd94f8b4 f2 19377bff55719a6ed.pdf?sfvrsn=ebe78315_6 (accessed 2020 Apr. 18).
19. Viera AJ, Garrett JM. Understanding interobserver agreement: the kappa statistic. Fam Med 2005;37:360-3.

20. Foster ED, Deardorff A. Open Science Framework (OSF). 7 Med Libr Assoc 2017;105. doi: 10.5195/jmla.2017.88

21. Sharing research data and findings relevant to the novel coronavirus (COVID-19) outbreak [news release]. London (UK): Wellcome Trust; 2020 Jan. 31. Available: https://wellcome.org/coronavirus-covid-19/open-data (accessed 2020 Oct. 28).

22. Williams DR, Cooper LA. COVID-19 and health equity: a new kind of "herd immunity.". 7AMA 2020;323:2478-80.

23. Haynes N, Cooper LA, Albert MA; Association of Black Cardiologists. At the heart of the matter: Unmasking and addressing the toll of COVID-19 on diverse populations. Circulation 2020;142:105-7.

24. Berger ZD, Evans NG, Phelan AL, et al. COVID-19: control measures must be equitable and inclusive. BM7 2020;368:m1141.

Affiliations: Department of Emergency Medicine (Hohl, Taylor, Andolfatto, Ting, Brar, Stachura), University of British Columbia; Centre for Clinical Epidemiology \& Evaluation (Hohl), Vancouver Coastal Health Research Institute, Vancouver, BC; Department of Pediatrics (Rosychuk), University of Alberta, Edmonton, Alta.; Department of Emergency Medicine (McRae), Foothills Medical Center, Calgary, Alta.; Department of Emergency Medicine (Brooks), School of Medicine, Queen's University; Kingston Health Sciences Centre (Brooks), Kingston, Ont.; Department of Family Medicine and Emergency Medicine (Archambault), Faculty of Medicine, Université Laval; VITAM (Centre de recherche en santé durable) (Archambault, Mercier), Québec, Que.; Division of EMS (Fok, Dahn, Wiemer), Department of Emergency Medicine, Dalhousie University; Charles V. Keating Emergency and Trauma Centre (Fok, Dahn, Wiemer), QEII Health Sciences Centre, Halifax, NS; Department of Emergency Medicine (Davis), College of Medicine, University of Saskatchewan, Saskatoon, Sask.; Department of Emergency Medicine (Jelic), Rady Faculty of Health Sciences, University of Manitoba, Winnipeg, Man.; Emergency Department (Turner), Jewish General Hospital; Department of Emergency Medicine (Turner), Faculty of Medicine and Health Sciences, McGill University, Montréal, Que.; Department of Emergency Medicine (Rowe, Hayward, Khangura), Faculty of Medicine \& Dentistry, and School of Public Health (Rowe), University of Alberta, Edmonton, Alta.; Centre de recherche (Mercier), CHU de Québec, Université Laval, Québec, Que.; Sunnybrook Health Sciences Centre (Cheng, Atzema); Division of Emergency Medicine (Cheng, Landes, Vaillancourt, Morrison), Department of Medicine, Faculty of Medicine, University of Toronto, Toronto, Ont.; Emergency Department (Taylor), Royal Columbian Hospital, New Westminster, BC; Départements de médecine de famille et de médecine d'urgence (Daoust), Faculté de médecine, Université de Montréal; Hôpital du Sacré-Coeur-de-Montréal (Daoust), CIUSSS Nord-de-l'ile-de-Montréal, Montréal, Que.; Department of Emergency Medicine (Ohle), Health Sciences North; Northern Ontario School of Medicine (Ohle), Sudbury, Ont.; Lions Gate Hospital (Andolfatto, Stachura), North Vancouver, BC; ICES Central (Atzema); University Health Network (Landes), Toronto, Ont.; Department of Emergency Medicine (Lang), Cumming School of Medicine, University of Calgary; Rockyview General Hospital (Lang), Calgary, Alta.; Department of Family Practice (Martin), Faculty of Medicine, University of British Columbia, Vancouver, BC; Abbotsford Regional Hospital \& Cancer Agency (Martin), Abbotsford, BC; Department of Emergency Medicine (Mohindra), North York General Hospital, North York, Ont.; Department of Emergency Medicine (Vaillancourt, Morrison), St. Michael's Hospital, Unity Health Toronto, Toronto, Ont.; Division of Emergency Medicine (Welsford), Department of Medicine, McMaster University; Hamilton Health Sciences (Welsford), Hamilton, Ont.; Surrey Memorial Hospital (Brar), Surrey, BC; Department of Emergency Medicine (Yadav, Perry), University of Ottawa; Ottawa Hospital Research Institute (Yadav, Perry), Ottawa, Ont.; Division of Emergency Medicine (Yan), Department of Medicine, London Health Sciences Centre; Schulich School of Medicine and Dentistry (Yan), Western University, London, Ont.; BC SUPPORT Unit (McGavin), Vancouver, BC

Contributors: Corinne Hohl, Laurie Morrison, Andrew McRae, Steven Brooks, Patrick Archambault, Patrick Fok, Philip Davis, Tomislav Jelic, Rhonda Rosychuk, Jeffrey Perry and Éric Mercier initiated the network, designed the registry and secured funding to conduct the study. Rhonda Rosychuk supervised data analysis, and all authors interpreted study findings and will contribute to initiating new manuscripts. Corinne Hohl and Laurie Morrison drafted the manuscript, and all authors have reviewed, 
edited and approved the manuscript. All authors take full responsibility for the manuscript.

Funding: Corinne Hohl is supported by a Michael Smith Foundation for Health Research Health Professional Investigator Award. All operating funds for the network were received from nonprofit organizations, including the national Canadian Institutes of Health Research (CIHR, 447679), BC Academic Health Science Network Society, BioTalent Canada, Ontario Ministry of Colleges and Universities (C-655-2129), the Saskatchewan Health Research Foundation (5357) and the Fondation CHU de Québec (Octroi No. 4007).

Content licence: This is an Open Access article distributed in accordance with the terms of the Creative Commons Attribution (CC BY-NC-ND 4.0) licence, which permits use, distribution and reproduction in any medium, provided that the original publication is properly cited, the use is noncommercial (i.e., research or educational use), and no modifications or adaptations are made. See: https://creativecommons.org/licenses/ by-nc-nd/4.0/

Data sharing: For investigators who wish to access Canadian COVID-19 Emergency Rapid Response Network data, proposals may be submitted to the network for review and approval by the network's peer-review publication committee, the data access and management committee and the executive committee, as per the network's governance. Information regarding submitting proposals and accessing data may be found at https://canadiancovid19ednetwork.org/.

Acknowledgements: The authors acknowledge Dr. Erica Lau, Maeve Wickham and Serena Small, who drafted the first version of this manuscript. Creating the largest network of collaboration across Canadian emergency departments in such a short period of time would never have been feasible without the tireless efforts of emergency department chiefs across the country, and of the research coordinators, assistants and trainees at participating sites who have contributed to this work. The authors thank Lisanne Boon (University of British Columbia, University Industry Liaison Office), Joleen Wright (Vancouver Coastal Health Research Institute Privacy), Pia Ganz (University of British Columbia Research Ethics Review Board) and Steven Tam (Vancouver Coastal Health Legal), who worked tirelessly to help ensure the network got up and running. Finally, the authors thank all of their colleagues in medicine, nursing and all of the allied health professions who have been on the front lines of this pandemic, bravely facing the risks of COVID-19 to look after our fellow citizens and after one another. The authors dedicate this network to you.

Supplemental information: For reviewer comments and the original submission of this manuscript, please see www.cmajopen.ca/content/9/1/ E261/suppl/DC1. 